NASA Technical Memorandum 86335

NASA-TM-8633519850010650

\title{
EFFICIENT SELF-CONSISTENT VISCOUS-INVISCID SOLUTIONS FOR UNSTEADY TRANSONIC FLOW
}

JAMES T, HOWLETT

JANUARY 1985
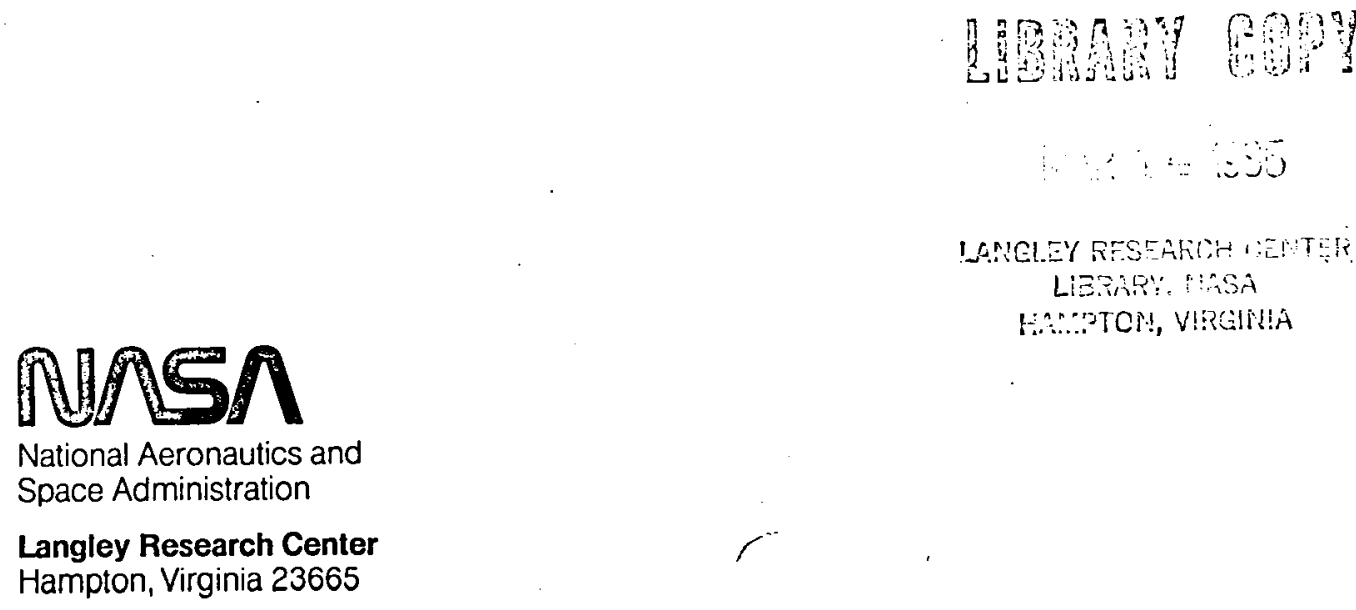

National Aeronautics and Langley Research Center Hampton, Virginia 23665 

NASA Technical Memorandum 86335

\section{EFFICIENT SELF-CONSISTENT VISCOUS-INVISCID SOLUTIONS FOR UNSTEADY TRANSONIC FLOW}

JAMES T, HOWLETT

JMNUARY 1985

\section{N/Sก


EFFICIENT SELF-CONSISTENT VISCOUS-INVISCID

SOLUTIONS FOR UNSTEADY TRANSONIC FLON

\author{
James T. Howlett \\ NASA Langley Research Center \\ Hampton, Virginia 23665
}

\section{Abstract}

An improved method is presented for coupling a boundary layer code with an unsteady inviscid transonic computer code in a quasisteady fashion. At each fixed time step, the houndary layer and inviscid equations are successively solved until the process converges. An explicit coupling of the equations is described which greatly accelerates the convergence process. Computer times for converged viscousinviscid solutions are about 1.8 times the comparable inviscid values. Comparison of the results ohtained with experimental data on three airfolls are presented. These comparisons demonstrate that the explicitly coupled viscous-inviscid solutions can provide efficlent predictions of pressure distributions and lift for unsteady two-dimensional transonic flows.

\section{Nomencl ature}

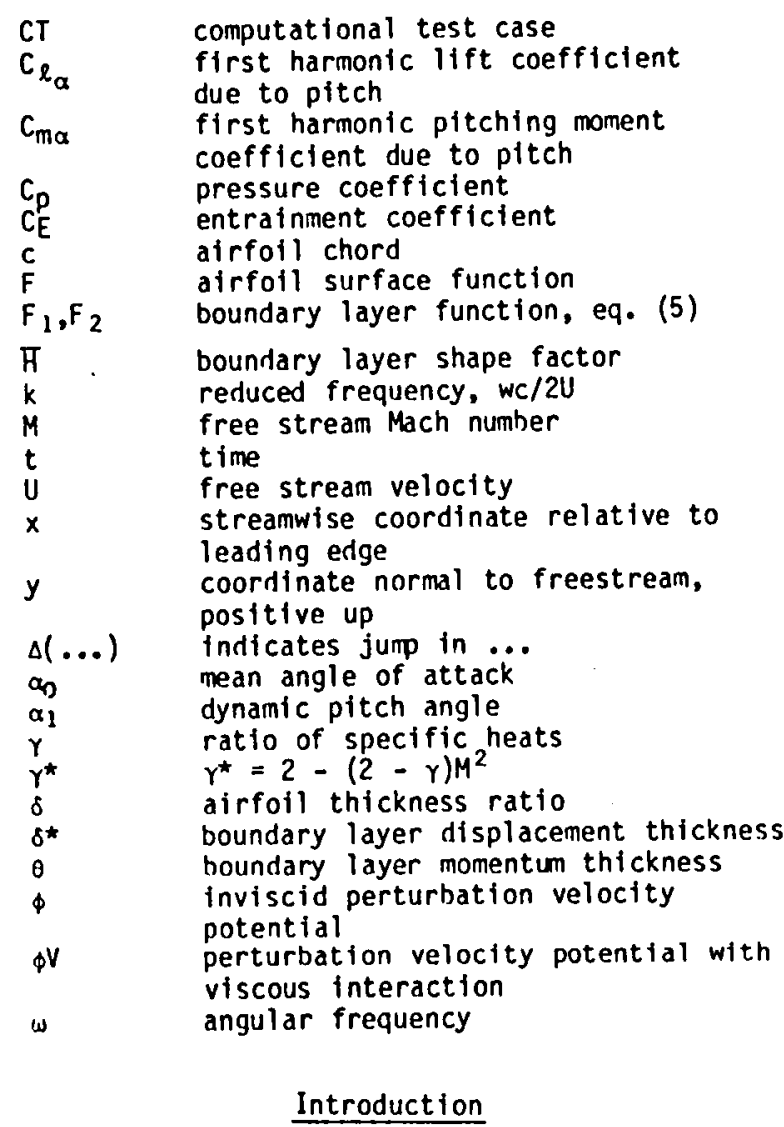

The importance of viscous effects in accurate predictions of steady and unsteady transonic aerodynamic loading is well-known. ' While Navier-Stokes computer codes can provide solutions $^{2}$ which include viscosity, their cost is prohibitive for routine use. As a result, extensive efforts are underway by various researchers to account for unsteady viscous effects by coupling a viscous boundary layer model with an otherwise inviscid analysis. 3-6 As commonly implemented, the inviscid outer flow solution provides the surface pressure distribution which is needed to solve the boundary layer equations. This yields the boundary layer thickness distribution which is used to modify the airfoll surface tangency boundary condition for the next iteration of the outer inviscid flow solution.

For steady flow problems, it has been demonstrated that this direct solution technique converges to consistent solutions of the houndary layer and inviscid equations which show good agreement with experiments ${ }^{7}$. These converged interactive solutions are referred to as self-consistent solutions. For unsteady flow problems, these iterative solution techniques are impractical due to the high computational expense associated with a large number of iterations.

Rizzetta' coupled the steady integral lag entrainment boundary layer model of $\mathrm{Green}^{8}$ with the LTRANR ${ }^{9}$ unsteady transonic code in a quasisteady manner using a non-iterative implicit technique. Guruswamy ${ }^{3}$ applied the method of Rizzetta in a study of viscous effects on oscillating airfoils with the result that up to 8000 time steps per cycle were required to obtain reasonably accurate answers. For step sizes even smaller than this the computer code became unstable. An alternative procenture was reported by Houwink ${ }^{4}$ who used an explicit method of viscous-inviscid coupling and obtained satisfactorily converged solutions with 120 time steps per cycle and computer times only $30 \%$ higher than the inviscid cases.

The present study began by incorporating Rizzetta's ${ }^{1}$ boundary layer algorith into the XTRAN2L code. 10 Several changes have been made to this procedure, the most important being the ability to iterate the viscous-inviscid solutions at each time step and the inclusion of an explicit coupling procedure. The boundary layer equations are numerically integrated from a specified transition point to the downstream boundary. The paper discusses the modifications which have been made to the computational algorith and presents comparisons of the resuits with experimental data on three airfoils.

\section{Analysis}

The inviscid core used in this study is the XTRAN2L computer code described hy whitlow. 10 This code is a modified version of the LTRAN2 code developed by Ballhaus and Goorjian. ${ }^{9}$ The 
XTRAN2L code solves the complete 2-D transonic small disturbance (TSD) equation given hy equation (1) and includes nonreflecting far-field boundary conditions.

$$
\begin{aligned}
& \frac{4 k^{2} M^{2}}{\delta^{2 / 3}} \phi_{t t}+\frac{4 k M^{2}}{\delta^{2 / 3}} \phi_{x t} \\
& =\left[\left(1-M^{2}\right) / \delta^{2 / 3}-M^{2}\left(\gamma^{\star}+1\right) \phi_{x}\right]_{x x}+\phi_{y y}
\end{aligned}
$$

The disturbance velocity potential $\phi$ is normalized by $\mathrm{CU}^{2 / 3}, \mathrm{c}$ is the airfoil chord, $\delta$ is the airfoil thickness ratio, and $U$ is the freestream speed. The spatial coordinates, $x$ and $y / s$, and the time, $t$, are normalized hy $c$, $c / s^{1 / 3}$, and $\omega^{-1}$ respectively, where $\omega$ is the frequency of unsteady motion. The reduced frequency (based on semi-chord) $k=\omega c / 2 U, M$ $\gamma^{*}=2-(2-\gamma) M^{2}$, where $\gamma$ is the ratio of specific heats.

The boundary conditions on the airfoil and wake for the inviscin code are:

airfofl: $\phi_{y}^{ \pm}=F_{x}^{ \pm}+F_{t}^{ \pm}$

wake:

$$
\begin{aligned}
& \Delta \phi_{y}=0 \\
& \Delta\left(\phi_{x}+\phi_{t}\right)=0
\end{aligned}
$$

where the \pm refers to the airfoil upper or lower surface, the function $F(x, t)$ denotes the airfoil surface, and $\Delta(\ldots)$ indicates a jump in the bracketed quantity.

To account for the viscous houndary layer in a quasi-steady manner, equations (2) and (3) are modified as follows (see Houwink ${ }^{5}$ and Rizzetta ${ }^{1}$ ):

airfoil: $\phi_{y}^{ \pm}=F_{x}^{ \pm}+F_{t}^{ \pm}+\left(\frac{\delta^{*}}{\delta c}\right)_{x}^{ \pm}$

wake: $\quad \Delta\left(p_{y}\right)=\Delta\left(\frac{\delta^{*}}{\delta c}\right)_{x}$

where $\delta^{\star}$ is the boundary layer displacement thickness determined from Green's lag entrainment equations.

Equation (2a) is a direct extension of the airRizzettal to include the term $\mathrm{F}_{t}^{ \pm}$which accounts for the time dependence of the airfoil motion in the houndary conditions. Note that although equation (2a) is a comonly used form to describe analytically the inclusion of the boundary layer equations, the precise manner in which this equation is incorporated into the computational algorith can be a key feature of the efficiency and accuracy of the method (see ref. 11, for example). This feature is discussed further in the following section.

The displacement thickness, $\delta^{\star}$, is computed as. a function of the houndary layer shape factor $\mathrm{H}$ and the momentum thickness $\theta$, which are determined, together with the entrainment coefficient, $C_{E}$, from Green's lag entrainment equations:

$$
\begin{aligned}
& \theta_{x}=f_{1}+f_{2} \phi_{x x} \\
& H_{x}=f_{3}+f_{4} \phi_{x x} \\
& \left(C_{E}\right)_{x}=f_{5}+f_{6} \phi_{x x}
\end{aligned}
$$

where the coefficients $f_{1}$ to $f_{6}$ are functions of $\theta, \vec{H}, C_{E}$, and other parameters which are described in detail in references 1 and 8 .

\section{Computational Procedure}

The basic algorithms for the viscous calculations are similar to those of Rizzetta 1 , where additional details may be found. Several modifications to the procedures of reference 1 have been incorporated into the present computer code and these modifications are enumerated in the following paragraphs.

1. In the original algorithm, the viscous equations are integrated using a Runge-Kutta algorithm given the value of $\left(C_{p}\right)_{x}$ at each outer flow grid point on the airfoil and wake. The integration spatial step size was found tn be too coarse and a finer step size is used for integration of the boundary layer equations. Ten houndary layer grid points are used between each pair of outer flow grid points. The required values of $\left(C_{p}\right)_{x}$ at the refined viscous grid points are determined by interpolation.

2. The empirical viscous wedge was eliminated, and integration of the houndary layer equations begins at a specified transition point. This allows a consistent description of the boundary layer from the specified transition point to the downstream boundary. Green's lag entrainment equations are simply integrated through the shock with no adverse effects. For results in this paper, transition is fixed at $10 \%$ chord unless stated otherwise.

3. Boundary layer smoothing was introduced to reduce instabilities. The values of $\phi_{x}$, $\Phi_{x x}$ used in the boundary layer calculations as weil as the values of $\delta^{*}$ and $\delta_{x}{ }^{*}$ are smoothed by averaging the values at $3-5$ adjacent grid points.

4. An option was added to allow for iterating the viscous-inviscid solutions at each time step. In Rizzetta's analysis, 1 the last term on the right of equation (2a) is implemented as:

$$
\begin{aligned}
\left(\frac{\delta^{*}}{\delta c}\right)_{x}= & F_{1}+F_{2} \phi_{x x} \\
& =F_{1}+\frac{2 F_{2}}{x_{i+1}-x_{i-1}} \\
& \cdot\left[\left(\frac{\phi_{i+1, j}^{n}-\phi_{i, j}^{n+1}}{x_{i+1}-x_{i}}\right)-\left(\frac{\phi_{i, j}^{n+1}-\phi_{i-1, J}^{n+1}}{x_{i}-x_{i-1}}\right),\right.
\end{aligned}
$$

where $F_{1}$ and $F_{2}$ are functions described in reference 1. Now $\phi_{j+1, J}^{n}$ and $\phi_{i, j}^{n+1}$ in this equation implicitly couple the two time levels $n$ and 
$n+1$ at the two streamwise stations $x_{i+1}$ and $x_{i}$. In the present analysis, these implicit equations are iterated at each time step.

Figure la presents a flow chart which describes the iteration procedure. For $t=t_{n+1}$ the boundary layer functions $F_{1}, F_{2}$ are calculated using the potential solution $\phi^{n}$ from the previous time step. During the $y$-sweep those, functions are used in eq. (5) to implicitly couple the boundary layer with the inviscid outer flow. The resulting solution for the coupled potential is denoted hy $\phi V^{n+1}$. This coupled solution, $\phi V^{n+1}$, is then used to re-calculate the boundary layer parameters and the process is repeated. Most applications investigated have required 5-25 iterations for convergence.

5. The coupling between the viscous houndary layer and the inviscid core was made explicit. This alternate approach to viscous-inviscid coupling is based upon the work of Houwink. ${ }^{4}$ The explicit coupling between the boundary layer and the inviscid solution on the airfoil (but not on the wake) is implemented by the direct use of equation (2a) in the expression for the airfoll downwash. That is, at time level $t=t_{n+l}$, the last term on the right of equation (2a) is evaluated by using values at the previous time step $t=t_{n}$ as follows:

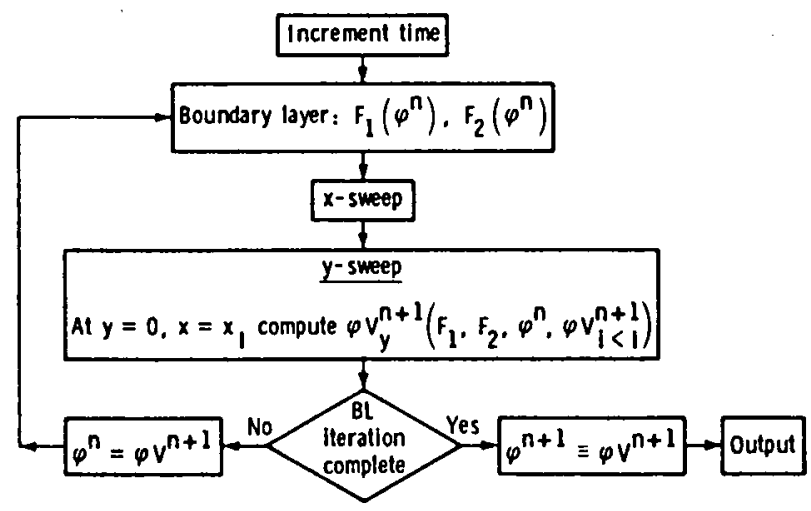

a) ORIGINAL VISCOUS COUPLING

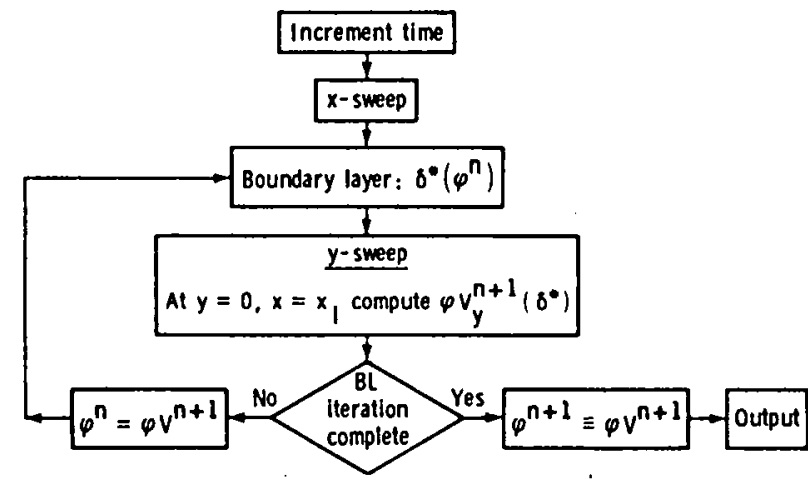

b) EXPLICIT VISCOUS COUPLING

Fig. 1 Flow charts for houndary layer coupling iterations. $\left(\frac{\delta^{*}}{\delta c}\right)_{x}=\frac{1}{\delta c}\left[\frac{\delta_{{ }_{i}^{*}}^{n}-\delta^{*}{ }_{i-1}^{n}}{x_{i}-x_{i-1}}\right]$

This procedure does involve a lag of boundary layer displacement thickness by one time step, but it does not involve an implicit coupling between different time levels at different streamwise stations.

A flow chart which indicates the explicit coupling algorittm is shown in figure 1b. As the figure indicates, the modifications for the explicit viscous coupling are included during the $y$-sweep of the alternating-direction-implicit (ADI) solution. The $x$-sweep calculations are identical for both forms of coupling. For computational efficiency, the self-consistent iterations are only done over the $y$-sweep. In practice, converged self-consistent solutions have been obtained in one iteration for most cases, resulting in more accurate viscous-inviscid solutions with reasonable computer times.

\section{Results and Discussion}

Convergence Studies for Original Coupling

In order to test the validity of the self consistent iteration procedure as described by modifications 1 through 4 , extensive calculations have been performed on several airfoils. The results presented in this section are for the NACA 64A010 airfoil (theoretical section). This airfoil, at a Mach number of 0.78 and one degree angle of attack has a moderately strong shock near midchord that is typical of the cases of interest. The results obtained for this airfoil are similar to the results obtained for the other airfoils studied.

The calculation procedure was as follows: Steady results for an inviscid analysis were calculated and used as a starting solution for a steady viscous solution. The steady viscous solution was then used as a starting solution for the unsteady viscous calculations. Innless otherwise stated, the unstearly results presented herein were run at 360 time steps per cycle and transient effects were sufficiently damped out after two cycles to obtain the harmonic components by a Fourier analysis. The number of viscous iterations per time step was varied and various unsteady quantities such as pressure, $1 i \mathrm{ft}$, and moment were compared as the number of iterations was increased. Comparisons with the results of Guruswamy ${ }^{3}$ indicate that the effect of these iterations is closely equivalent to taking smaller time steps. That is, 360 time steps per cycle with two iterations per time step gives results similar to using 720 time steps per cycle. However, the fterative procedure does converge, as will be shown below, whereas the use of successively smaller time steps can lead to stability problems ${ }^{3}$.

Fig. 2 shows pressure distributions on the airfoil upper surface for steady flow at one degree angle of attack and 0.78 Mach number. As the figure indicates, a moderately strong shock is located near midchord with the viscous shock location slightly upstream of the inviscid shock. 


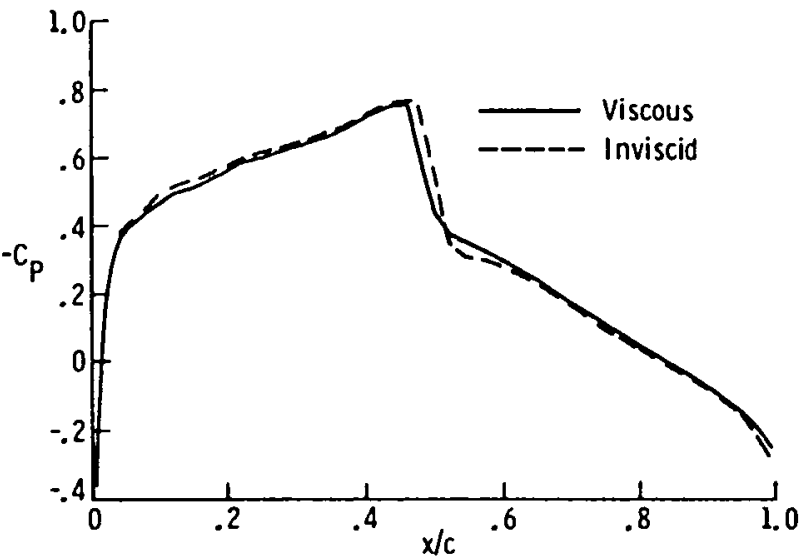

Fig. 2 Upper surface steady pressure for MACA 64A010 (theoretical) airfoll: original viscous coupling, $a_{0}=1^{\circ}, M=0.78$

The first harmonic components of unsteady pressure distributions for one degree oscillation in pitch $\left(\alpha_{1}=1^{\circ}\right)$ with $k=0.1$ for varying numbers of iterations are shown in Fig. 3. For one iteration (Fig. $3 a$ ), the viscous solution is quite different from the inviscid solution. For five iterations (Fig. 3b), the viscous and inviscid solutions are nearly coincident except in the vicinity of the shock. Similar to results for the steady solution, the unstearly viscous solution has a somewhat weaker shock wave at a slightly upstream location. Comparison of Figs. $3 b$ and $3 c$ shows that the viscous solution after 10 iterations is nearly identical to the solution with five iterations except for some minor differences in magnitude near the shock. This clearly demonstrates the importance of obtaining converged self-consistent solutions in assessing the effects of viscosity on pressure distributions.

Fig. 4 shows the real and imaginary parts of the first harmonic component of the unsteady lift and moment as the number of iterations per time step is varied. For both $11 \mathrm{ft}$ and moment, the real part of the coefficient changes significantly as the number of iterations is increased from one to five, thereafter settling down and asymptotically approaching the converged value. The moment coefficient changes sign between the second and third iterations before asymptotically approaching the converged value. Since the moment coefficient is calculated about the quarter-chord for this case, some of this variation may be due to the sensitivity of the coefficient to small changes in the flow. Although not shown on this figure, calculations have been carried out for up to 22 iterations per time step and the results were identical to those obtained for 10 iterations. The imaginary part of the unsteady lift changes very little during the iterations whereas the trend for the imaginary part of the unsteady moment is quite similar to that of the real part. Also shown on the figure are the corresponding results for the inviscid analysis. The converged solution indicates that for this case the effects of viscosity are very minor and primarily result in a small reduction in the magnitude of hoth the unsteady lift and moment.

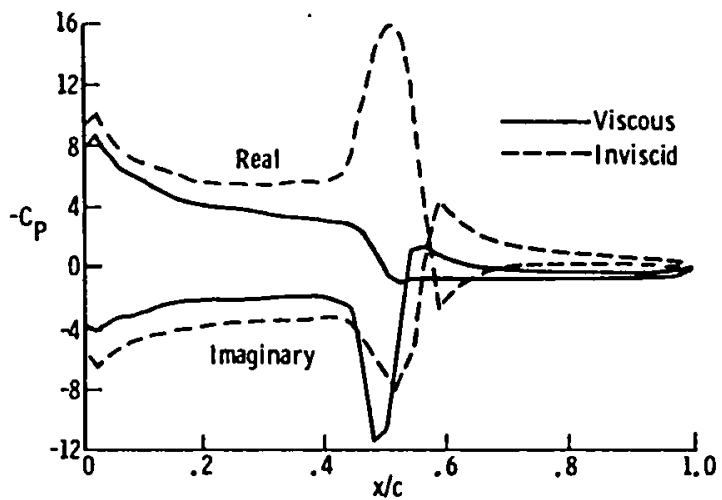

a) 1 VISCOUS ITERATION

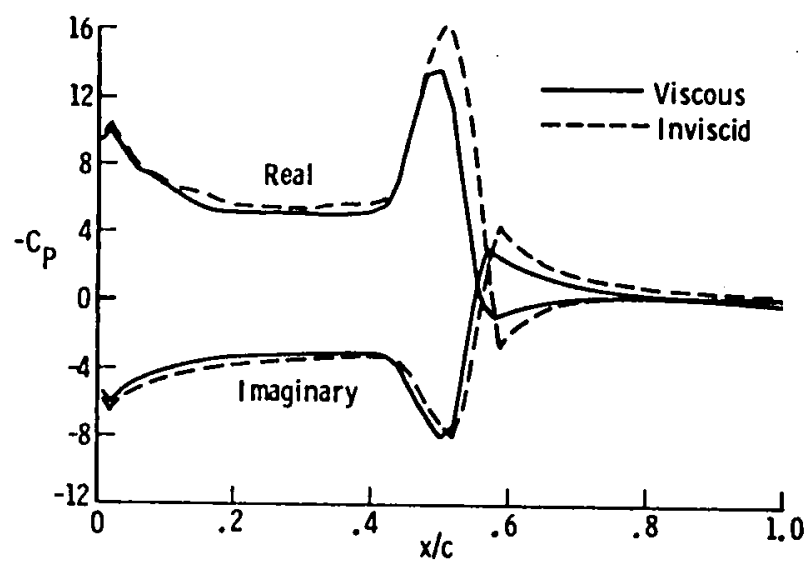

b) 5 VISCOUS ITERATIONS

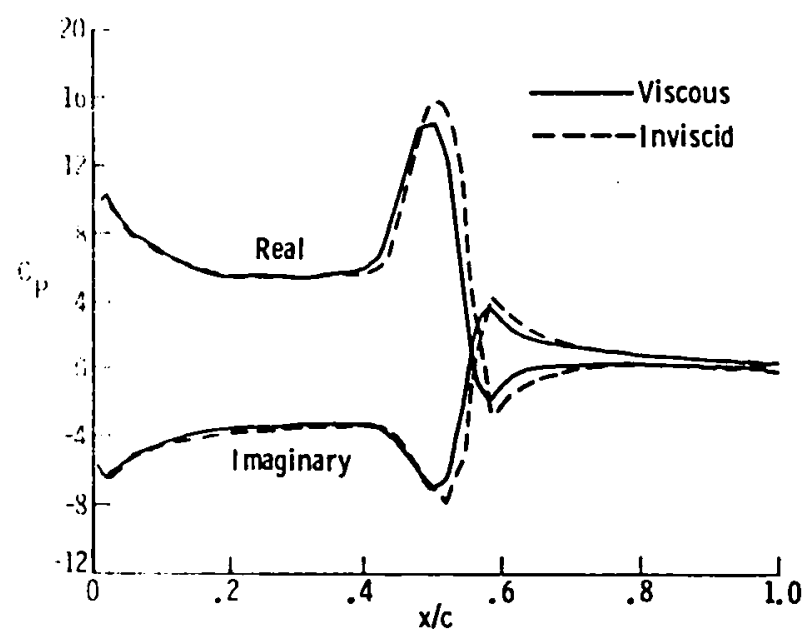

c) 10 VISCOUS ITERATIONS

Fig. 3 Effect of number of viscous iterations for original viscous coupling method on upper surface unsteady pressure for MCA 64A010 (theoretical) airfoil: $a_{0}=1^{\circ}, a_{1}=1^{\circ}, M=0.78$, $k=0.1$. 


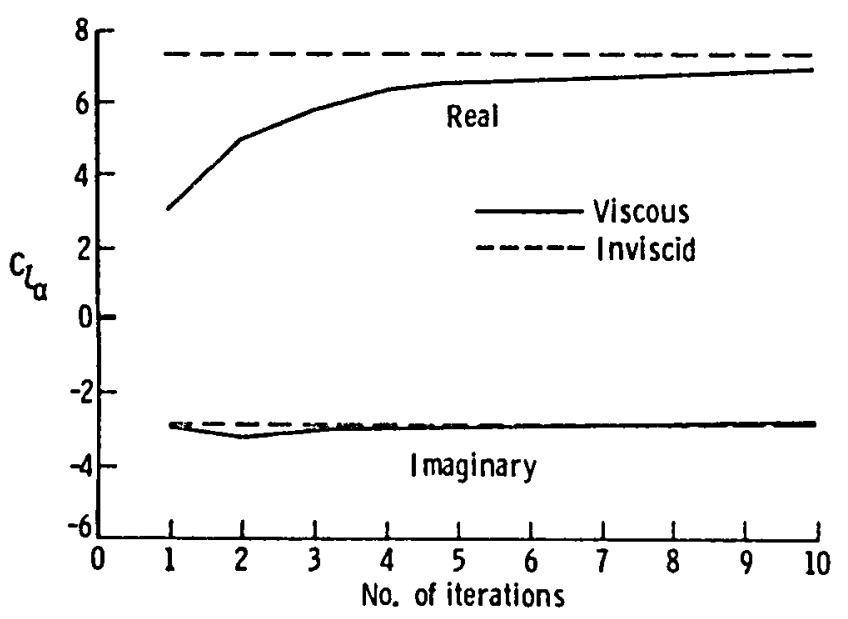

a) UASTEADY LIFT

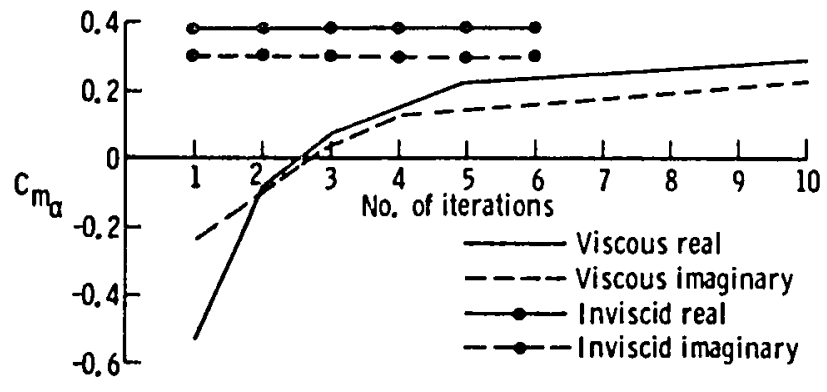

b) UNSTEAOY MOMENT

Fig. 4 Effect of number of viscous iterations for original viscous coupling method on unsteady forces for MACA $64 A 010$ (theoretical) alrfoil: $a_{n}=1^{\circ}, \alpha_{1}=1^{\circ}, M=0.78, k=0.1$.

\section{Explicit Coupling Studies}

The computer costs associated with the large number of iterations required for converged self-consistent solutions with the original formulation of viscous-inviscid coupling is quite high. Each viscous iteration increases the computer time hy ahout $80 \%$ of the inviscid solution time. A practical alternative for reducing the number of iterations has been found to be the explicit coupling between the inviscid equations and the houndary layer displacement thickness described previously in modification number 5. The explicftly coupled equations compute a self-consistent solution with very few iterations, usually just one. Hence, explicit coupling results in converged self-consistent solutions in computer times which are about 1.8 times the comparable tnviscid values in many cases.

Fig. 5 shows the first harmonic of the unsteady pressure distribution on the upper surface of the NACA 64A010 airfoil at one degree angle of attack. Mach number of $0.78, \alpha_{1}=1^{\circ}$, and $k=0.1$ as computed using the explicit boundary layer coupling. Comparison of Fig. 5a with Fig. $3 c$ shows that the pressures ohtained with one iteration of the explictt coupling are practically identical to those from the original coupling with 10 iterations. As shown hy Fig.
$5 b$, the results for 10 iterations with explicit coupling are the same as those obtained with one iteration, thus demonstrating that this solution is in fact a converged self-consistent solution.

\section{Comparison with Experiments}

In this section calculations from the present analysis with the explicit viscous-inviscid coupling are compared with experimental data for several of the computational test cases selected by the AGARD Structures and Materials Panel 12 as well as some published data for the MBB-A3 airfoll. I3 Additional information on the experimental configurations may be found in the references. ${ }^{12}, 13$

NACA 64A010. The experimental results presented here are for the model tested at the NASA Ames Research Center and are taken from Chapter 2 of reference 12. Fig. 6 shows steady pressure distributions on the airfoil lower surface for the inviscid, viscous and experimental results.

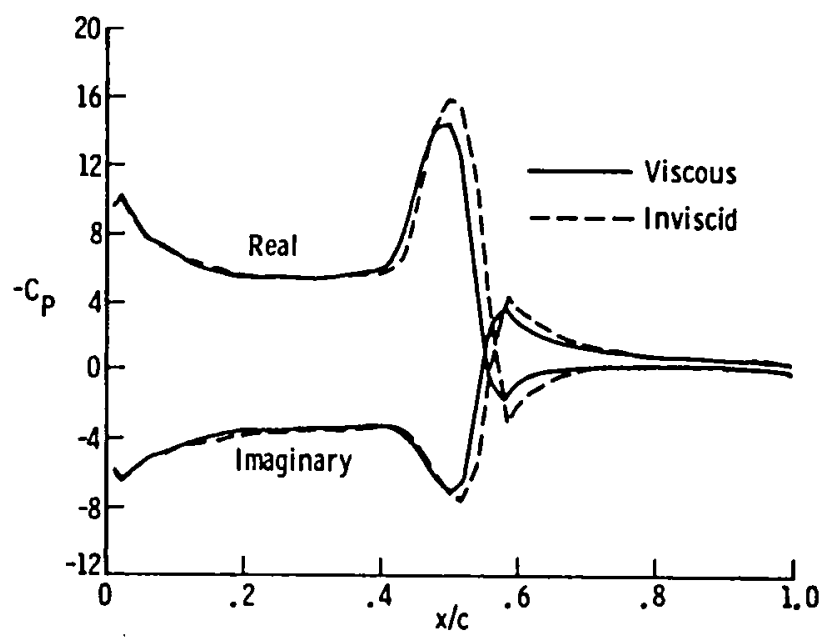

a) 1 VISCOUS ITERATION

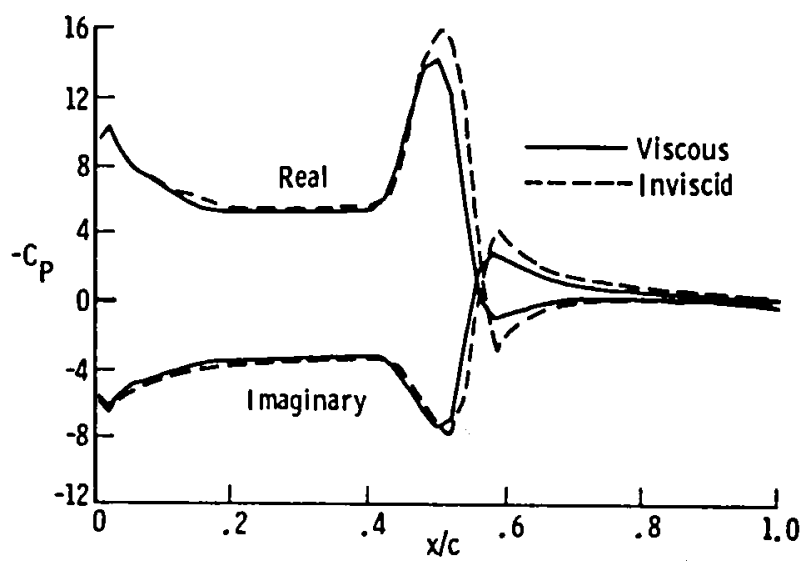

b) 10 VISCOUS ITERATIONS

Fig. 5 Unsteady upper surface pressure with explicit viscous coupling compared with inviscid results for MACA $64 A 010$ (theoretical) airfoll: $\infty_{0}=10, \alpha_{1}=1^{\circ}, k=0.1$. 
The Mach number is 0.796 and the angle of attack is -0.21 degrees. These steady results correspond to AGARD Computational Test (CT) case $5^{12}$. As for the previous results, the viscous effects are small. The predicted shock location for the viscous analys is is slightly forward of the inviscid shock location and agrees well with the experimental result. Downstream of the shock, both the viscous and inviscid analyses agree fairly well with the experiment.

Fig. 7 presents unsteady pressure distributions on the lower surface of the airfoll for AGARD CT case 5. The first harmonic components are compared with the experimental values for $a_{1}=1^{\circ}$ and $k=0.101$. The viscous shock pulse is about two percent chord upstream of the inviscid shock pulse and correlates better with the experimental values.

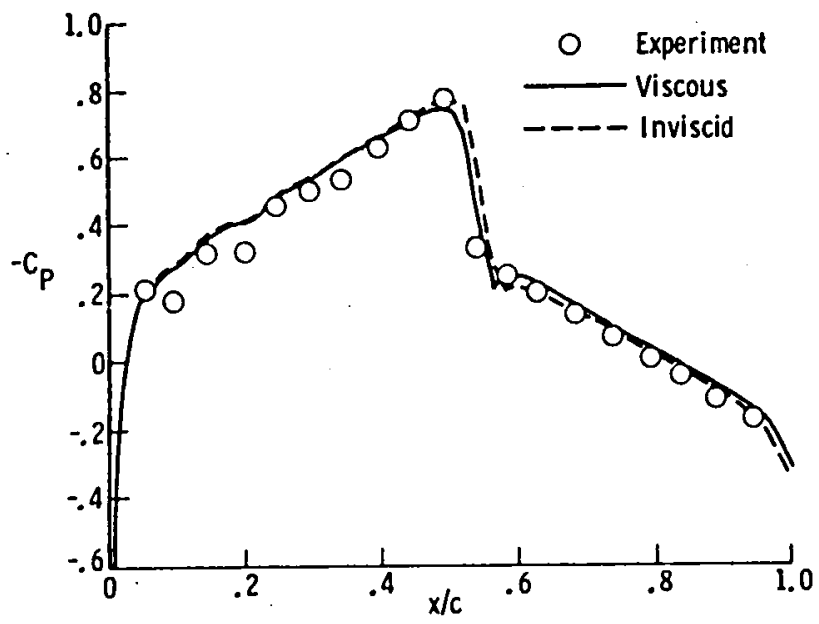

Fig. 6 Comparison between analytical and experimental steady pressure for lower surface of NACA 64A010 (experimental) airfoil: Explicit viscous coupling, on $=-0.21^{\circ}, M=0.796$ (ref. AGARD CT Case 5).

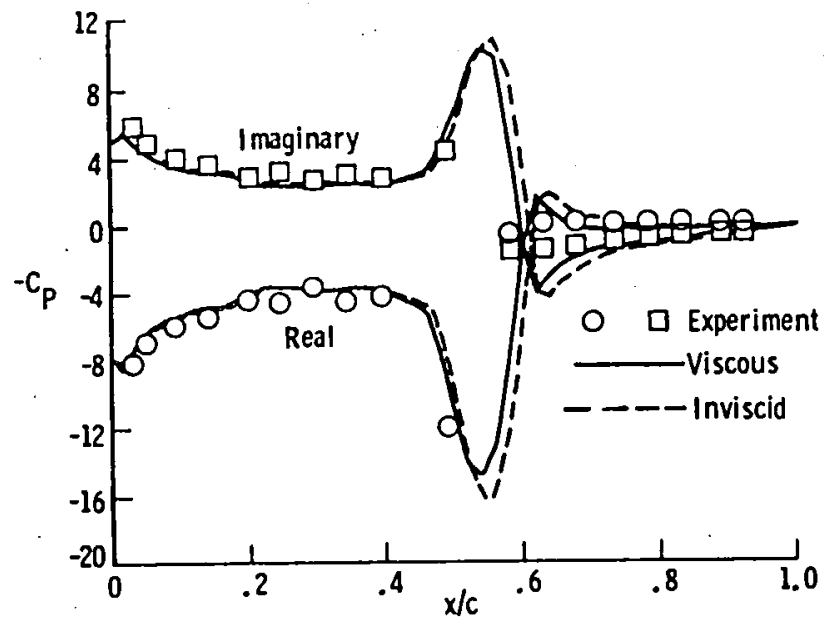

Fig. 7 Comparison between analytical and experinental unsteady pressure for lower surface of MACA 64A010 (experimental) airfoll: Explicit viscous coupling, 1 iteration, $a_{0}=-0.21^{\circ}$, $\alpha_{1}=1^{\circ}, M=0.796, k=0.101$ (AGARD CT Case 5).

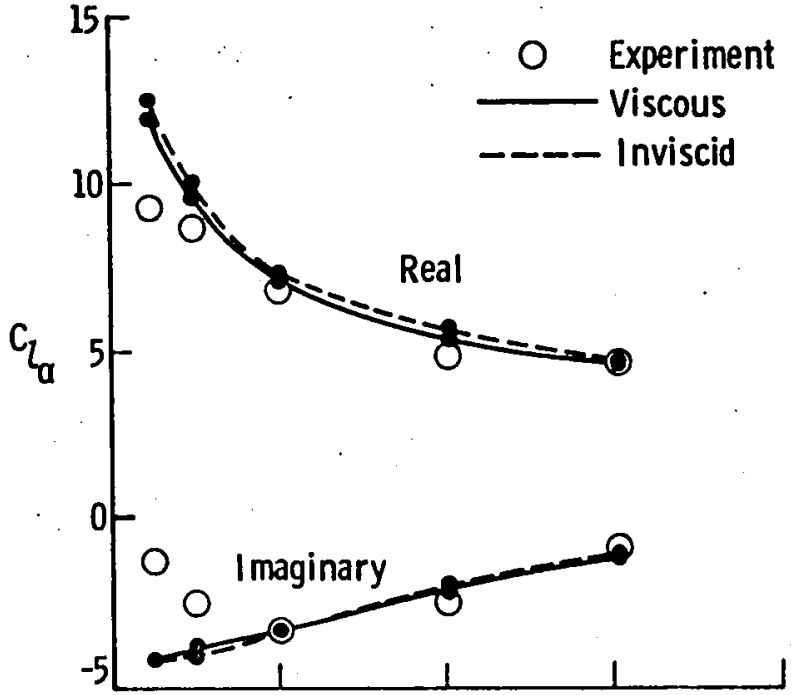

a) UNSTEADY LIFT

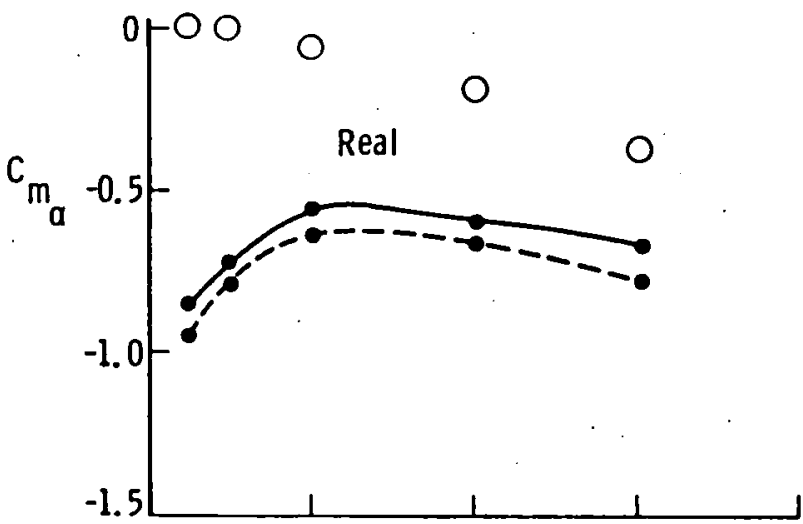

b) REAL PART OF UNSTEADY MOMENT

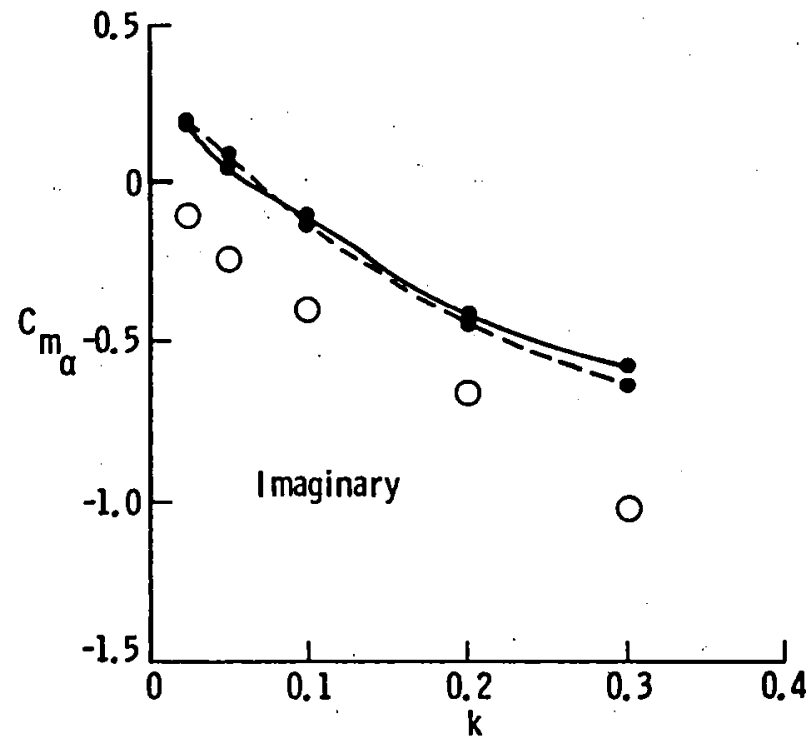

c) IMAGIMARY PART OF UNSTEADY MOMENT

Fig. 8 Unsteady forces versus reduced frequency for MACA $64 A 010$ (experimental) airfoil. 
In Fig. 8, unsteady $11 \mathrm{ft}$ and moment coefficients versus frequency are presented. As show in Fig. 8a, the viscous solution for the lift coefficient is closer to the experimental results than the inviscid solution and, for reduced frequencles greater than 0.1 , agrees well with the experimental data. The calculated moment coefficients shown in Figs. $8 b$ and $8 c$ have the same trends as the experimental data although the actual values are significantly different. The source of this difference is unknown and further investigation is needer. The results do show that viscous effects are not the dominant effect in this difference.

For this airfoll, over the range of reduced frequencies investigated, the explicit coupling inethod provides efficient viscous predictions of unsteady pressure distributions and lift coefficients which agree better with experiment than the inviscid results.

MBB-A3. The experimental pressure distribution for the supercritical MBB-A3 airfoil for steady flow is taken from reference 13.

Fig. 9 presents comparisons of steady pressure distributions for the experiment, the vis cous and the inviscid analyses for the design condition of the airfoil. Note that the calculated values were obtained by using the actual experimental values for the Mach number and the angle of attack rather than values which have heen adjusted to match flow conditions in the wind tunnel as is frequently done in comparisons with this particular data. For the viscous solution, the shock is located about four percent chord forward of the inviscid shock location and is somewhat weaker. However, a large discrepancy between the experimental values and the viscous prediction still exists and further investigation is required. Possible explanations for this discrepancy include inadequate theoretical modeling of the shock-boundary layer interaction, trafling edge effects, or inadequate modeling of the flow conditions in the wind tunnel.

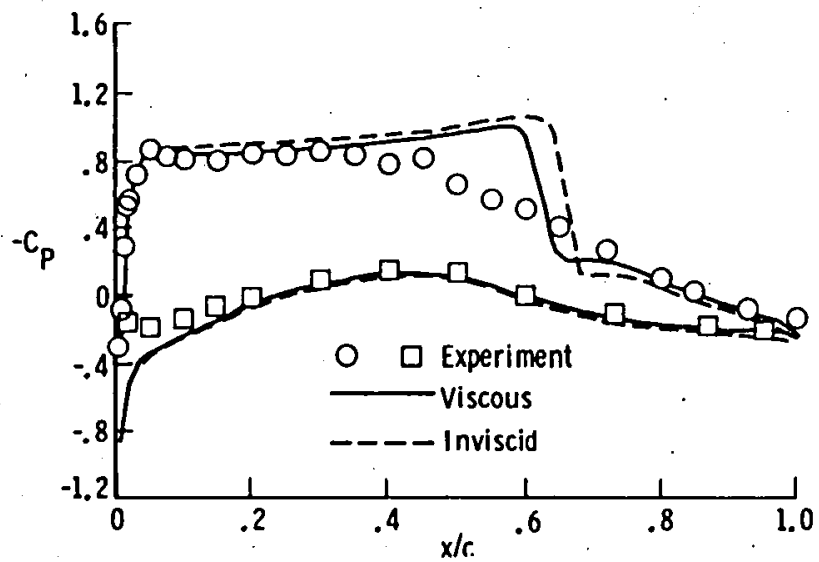

Fig. 9 Comparison of analytical and experimental steady pressure for supercritical MBB-A3 airfoil: explicte viscous coupling, $\infty_{0}=1.5^{\circ}$, $M=0.765$.
Fig. 10 presents the unsteady pressure distribution for the viscous and inviscid solutions for a reduced frequency of 0.1 and $\alpha_{1}=0.5^{\circ}$. The results are analogous to those for the steady case. On the upper surface of the airfoll, the shock location for the viscous solution is about five percent chord forward of the inviscid shock location and the shock strength for the viscous solution is considerably weaker than that of the inviscid solution. on the lower surface of the airfoil, the unstearly pressure distributions for both. Solutions are essentially the same.

NLR 7301. The experimental results for the supercritical NLR 7301 airfoil are taken from Chapter 4 of reference 12 . Calculated results presented herein correspond to the test conditions for AGARD CT cases 3 and $5^{12}$ al though some differences do exist between these values and actual wind tunnel test conditions. An excellent discussion of this point is given by Lambourne in Chapter 0 of reference 12 .

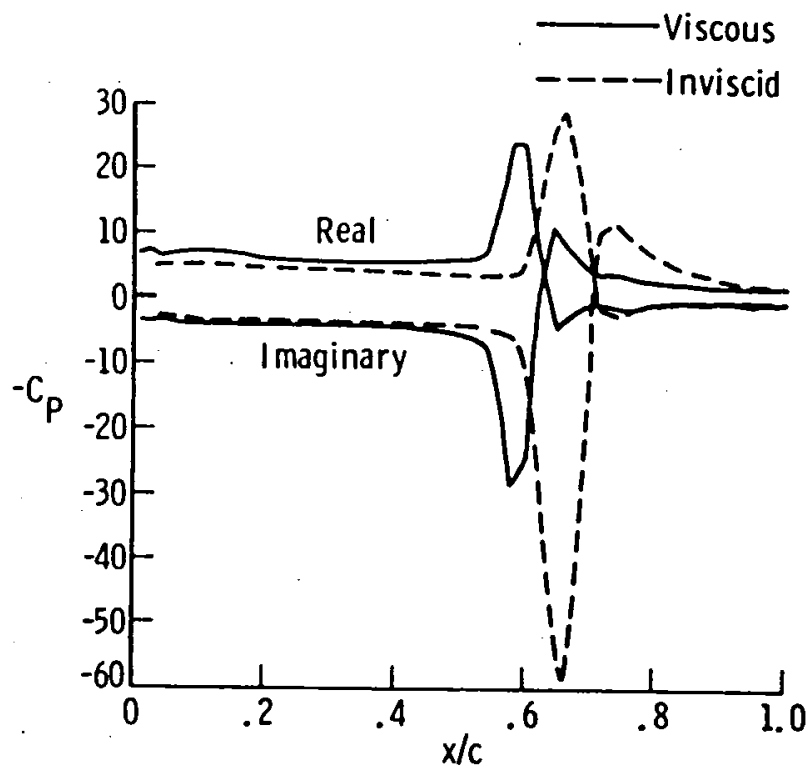

a) UPPER SURFACE PRESSURE

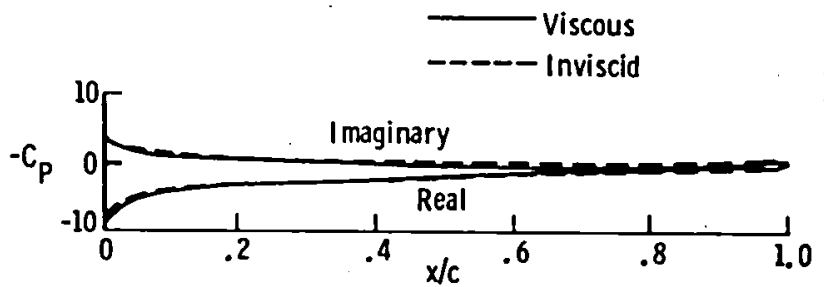

b) LOAER SURFACE PRESSURE

Fig. 10 Comparison of viscous and inviscid analytical unsteady pressure for supercritical MBB-A3 airfoil: explicit viscous coupling, $\alpha_{0}=1.5^{\circ}, \alpha_{1}=0.5^{\circ}, M=0.765, k=0.1$. 
Fig. 11 presents steady pressure distributions for experimental and analytical cases with $M=0.7$ and $a_{0}=2^{\circ}$. These values correspond to the AGARD $C T$ cases 3 and 5 . For the viscous solution, transition is fixed at $30 \%$ chord to correspond to the experimental configuration 12 . The shock location for the inviscid solution is about $20 \%$ chord downstream of the experimental shock location. The viscous solution has a shock location and strength nearly coincldent with the experimental result. On the airfoil lower surface, pressures from the viscous solution are slightly below the experimental values although they agree better with the experiment than the inviscid values. This close agreement between the experiment and the viscous solution for this difficult case may be somewhat fortuitous due to the previously mentioned differences between the parameters for the AGARD cases and the wind tunnel tests.

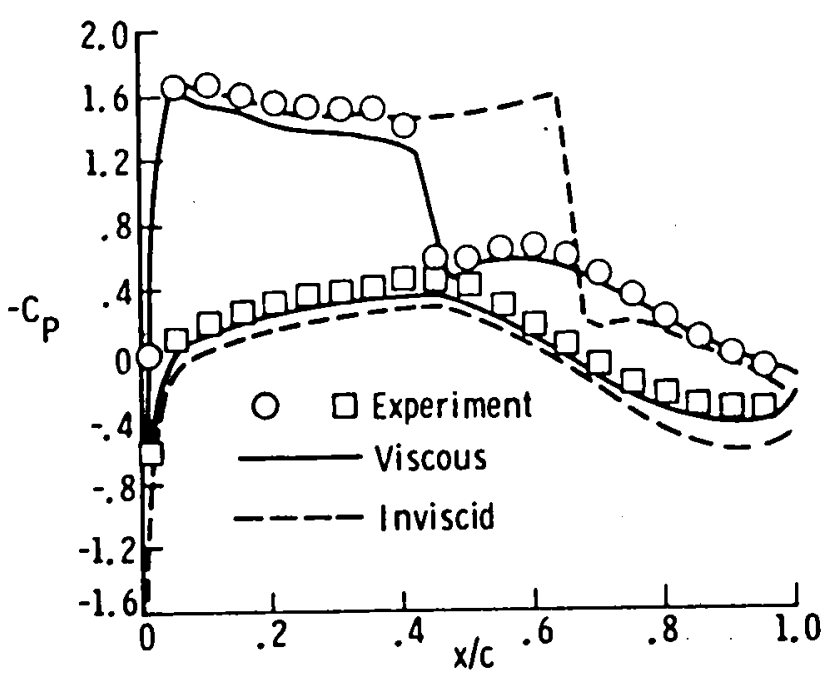

Fig. 11 Comparison of analytical and experimental steady pressure for supercritical MR 7301 airfoil: explicit viscous coupling, $\infty_{0}=2^{\circ}$, $M=0.7$ (ref. AGARD CT Case 3 and 5 ).

In Fig. 12, unsteady pressure distributions are plotted corresponding to AGARD CT case 3 with $\alpha_{l}=0.5^{\circ}$ and $k=0.072$. The unsteady vis cous results for this airfoil were computed with 720 time steps per cycle and two viscous iterations (explicit coupling) per time step. As Fig. 12a shows, on the alrfoll upper surface the viscous solution agrees much better with the experiment than the inviscid solution. The viscous shock pulse is silghtly downstream of the experimental location, significantly weaker and broader. As show in figure $12 \mathrm{~h}$, on the airfoil lower surface viscous effects are small and the agreement between calculated results and experiment is good.

Fig. 13 presents unsteady pressure distributions for a higher reduced frequency of $k=0.192$ with $\alpha_{1}=0.5^{\circ}$ (AGARD CT case 5). As with the previous case, the viscous solution agrees quite well with the experiment whereas the inviscid solution is considerably different. Fig. 13a shows that the location of the

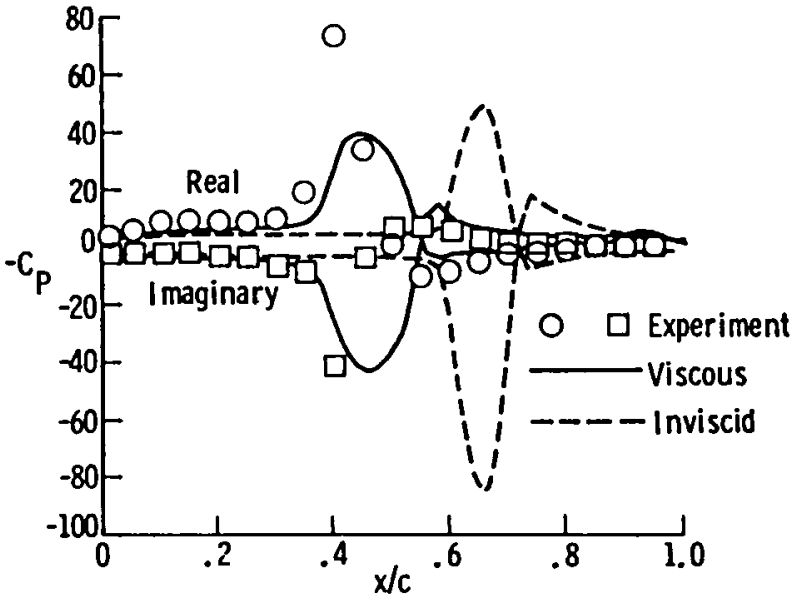

a) UPPER SURFACE PRESSURE

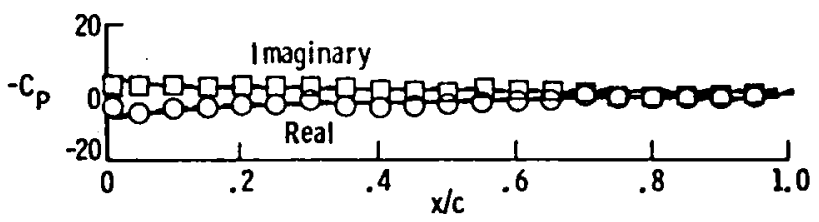

b) LONER SURFACE PRESSURE

Fig. 12 Comparison of analytical and experimental unsteady pressure for supercritical MR 7301 airfoil: explicit viscous coupling, 2 iterations, $a_{0}=2^{\circ}, a_{1}=0.5^{\circ}, M=0.07 k=0.072$ (NGARD Cो Case 3)

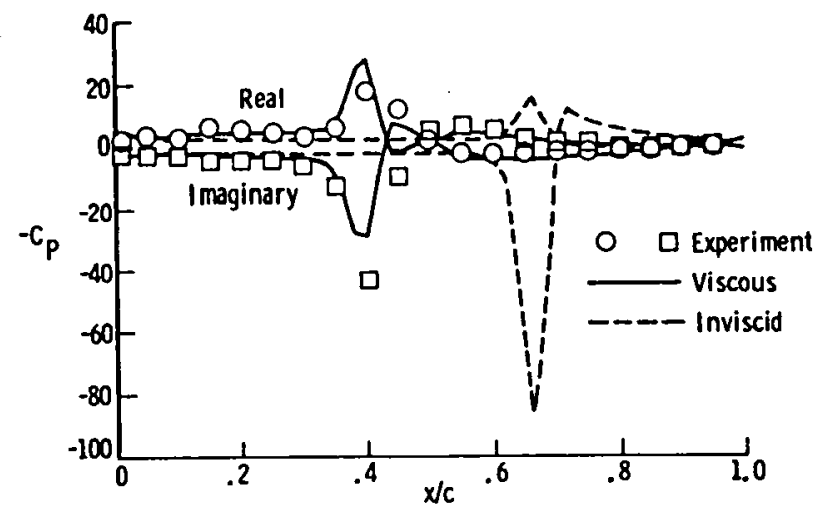

a) UPPER SURFACE PRESSURE

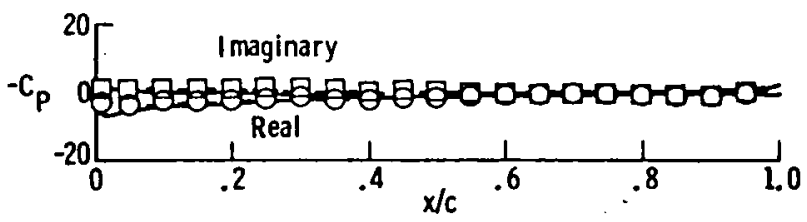

b) LOAER SURFACE PRESSURE

Fig. 13 Comparison of analytical and experimental unsteady pressure for supercritical MR 7301 airfoll: explicit viscous coupling, 2 iterations, of $=2^{\circ}, a_{1}=0.5^{\circ}, H=0.7, k=0.192$ (AgARD Cl Case 5). 
viscous shock pulse is correctly predicted although it is much narrower and some differences are noted in amplitude. On the airfoil lower surface, viscous effects are small and the calculated results agree well with the experiment as shown in Fig. $13 \mathrm{~b}$.

\section{Conclusions}

This paper has presented a study of selfconsistent solutions for viscous-inviscid interactions in unsteady two-dimensional transonic flow. Two different numerical techniques have been investigated for coupling the quasi-steady viscous boundary layer with the inviscid solution: 1) The original method of Rizzetta ${ }^{\text {l with }}$ the addition of self-consistent iterations; and 2) Explicit coupling based upon the work of Houwink ${ }^{4}$. Both methods are based upon the 2-D XTRAN2L inviscid transonic computer code coupled with a turbulent viscous boundary layer represented by Green's lag entrainment equations. In this study, the viscous wedge in Rizzetta's analysis has been eliminated and the transition point is specified. Each method successively solves the boundary layer and inviscid equations at each fixed time step until the process converges. The results demonstrate that both methods converge to self-consistent solutions. However, method 2, which incorporates explicitly coupled viscous-inviscid equations, computes a converged self-consistent solution in one or two iterations whereas method 1 requires 5 to 25 iterations to converge. The computer code with explicitly coupled equations yields computer times which are reduced by a factor of 20 from those of method 1. Accurate, self-consistent, converged, viscous-inviscid solutions are obtained in computer times that are about 1.8 times the comparable inviscid values.

Comparisons of unsteady forces and pressure distributions computed with the original coupling method for various numbers of iterations have demonstrated the importance of obtaining converged self-consistent solutions for an accurate assessment of viscous effects.

For the NACA $64 A 010$ (experimental) airfoil, unsteady lifting forces obtained by the viscous calculations with explicitly coupled boundary conditions agree well with experimental results for reduced frequencies greater than 0.1 over the range studied. Unsteady moment coefficients indicated the same trend as the experimental results although the magnitudes were significantly different.

Comparisons of steady pressure distributions for the supercritical MBB-A3 airfoil show that the viscous shock location (explicit coupling) is about $4 \%$ chord forward of the inviscid shock location although a large discrepancy still exists between the viscous results and the experiment. The source of this discrepancy is not known and further investigation is required.

For the supercritical NLR 7301 airfoil, comparisons of experimental results with viscous solutions computed by the explicitly coupled equations clearly demonstrate that this type of analysis can yield accurate predictions of both steady and unsteady pressure distributions for this difficult case. Although some differences in shock strength do exist for the conditions investigated, both steady and unsteady shock locations are predicted with good accuracy.

The results presented demonstrate that self-consistent viscous solutions computed with the explicit coupling algorithm can provide efficient predictions of pressure distributions and lift for unsteady transonic flow which correlate better, sometimes significantly better, with experimental values than the inviscid solutions.

\section{Acknowledgement}

The author would like to express his appreciation to Dr. Peter Goorjian of the Applied Computational Aerodynamics Branch, NASA Ames Research Center, for providing the original

\section{References}

1. Rizzetta, Donald P.: Procedures for the Computation of Unsteady Transonic Flows Including Viscous Effects. NASA CR 166249, Jan. 1982.

2. Chyu, W. J. and Ono, K.: Unsteady Transonic Flow Over Conventional and Supercritical Airfoils. AIAA Paper No. 83-0235, AIAA 2lst Aerospace Sciences Meeting, Reno, NV, Jan. 1983.

3. Guruswamy, P. and Goorjian, P. M.: Effects of Viscosity on Transonic Aerodynamic and Aeroelastic Characteristics of Oscillating Airfoils. AIAA Paper No. 83-0888, 24th AIAA/ASME/ASCE/AHS Structures, Structural Dynamics, and Materials Conference, Lake Tahoe, NV, May 1983.

4. Houwink, R.: Results of a New Version of the LTRAN2-NLR code (LTRANV) for Unsteady Viscous Transonic Flow Computations. NLR Report No. NLR TR 81078U, July 1981.

5. Houwink, R. and Veldman, A. E. P.: Steady and Unsteady Separated Flow Computations for Transonic Airfoils. AIAA Paper No. 84-1608. AIAA 17th Fluid Dynamics, Plasmadynamics and Lasers Conference, Snowmass, CO, June 1984.

6. Rizzetta, D. P. and Borland, C. J.: Numerical Solution of Three-Dimensional Unsteady Transonic Flow Over Wings Including Inviscid/Viscous Interactions. NASA CR 166561, Feb. 1984.

7. Street, Craig L.: Viscous-Inviscid Interaction for Transonic Wing-Body Configurations Including Wake Effects. AIAA Journal. Vol. 20, No. 7, July 1982.

8. Green, J. E.; Weeks, D. J. and Brooman, J. W. F.: Prediction of Turbulent Boundary Layers and Wakes in Compressible flow by a Lag-Entrainnent Method. RAE Reports and Memoranda No. 3791, Jan. 1973. 
9. Ballhaus, W. F. and Goorjtan, P. M.: Implicit Finite-Difference Computations of Unsteady Transonic Flows About Airfoils, AIAA Journal, Vol. 15, No. 12, Dec. 1977.

10. Whitlow, Woodrow, Jr.: XTRAN2L: A Program for Solving the General-Frequency Unsteady Transonic Small Disturbance Equation. NASA TM 85723, Nov. 1983.

11. Melnik, R. E.: Turbulent Interactions on Airfolis at Transonic Speed-Recent Developments. In: Computation of Viscous-Inviscid Interactions, AGARD CP 291, Feb. 1981.

12. Anon: Compendium of Unsteady Aerodynamic Measurements. AGARD Report No. 702, Aug. 1982.

13. Bucciantini, G.; Oggiano, M. S. and Onorato, M.: Supercritical Airfoil MBB-A3 Surface Pressure Distributions, Wake and Boundary Condition Measurements. In: Experimental Data Base for Computer Program Assessment, AGARD Advisory Report No. 138, May 1979. 


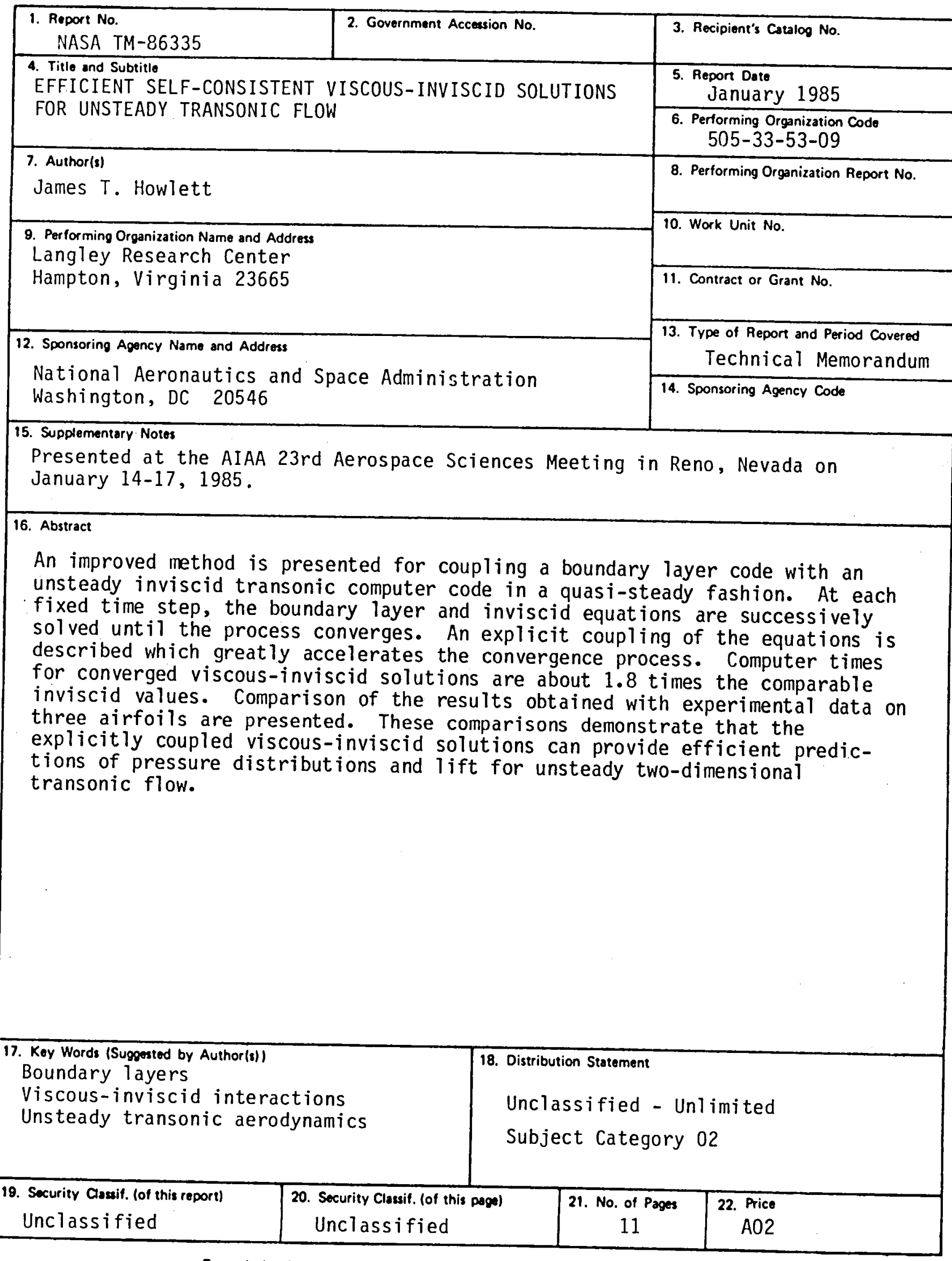


